\title{
SMOKING
}

\section{Clinical trial comparing nicotine replacement therapy (NRT) plus brief counselling, brief counselling alone, and minimal intervention on smoking cessation in hospital inpatients}

\author{
A Molyneux, S Lewis, U Leivers, A Anderton, M Antoniak, A Brackenridge, F Nilsson, \\ A McNeill, R West, J Moxham, J Britton
}

See end of article for authors' affiliations

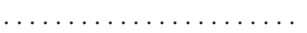

Correspondence to: Dr A Molyneux, Division of Respiratory Medicine, University of Nottingham, City Hospital, Hucknall Road, Nottingham NG5 1PB, UK:

andrew.molyneux@ nottingham.ac.uk

Accepted for publication 1 January 2003

\begin{abstract}
Background: Guidelines recommend that smoking cessation interventions are offered in all clinical settings to all smokers willing to make a quit attempt. Since the effectiveness of routine provision of behavioural counselling and nicotine replacement therapy (NRT) to smokers admitted to hospital has not been established, a randomised controlled trial of these interventions given together compared with counselling alone or minimal intervention was performed in hospital inpatients.

Methods: Medical and surgical inpatients who were current smokers at the time of admission were randomised to receive either usual care (no additional advice at admission), counselling alone (20 minute intervention with written materials), or NRT plus counselling (counselling intervention with a 6 week course of NRT). Continuous and point prevalence abstinence from smoking (validated by exhaled carbon monoxide <10 ppm) was measured at discharge from hospital and at 3 and 12 months, and self-reported reduction in cigarette consumption in smokers was assessed at 3 and 12 months.

Results: 274 inpatient smokers were enrolled. Abstinence was higher in the NRT plus counselling group $(n=91)$ than in the counselling alone $(n=91)$ or usual care $(n=92)$ groups. The difference between the groups was significant for validated point prevalence abstinence at discharge $155 \%, 43 \%$, $37 \%$ respectively, $\mathrm{p}=0.045)$ and at 12 months $(17 \%, 6 \%, 8 \%, p=0.03)$. The respective differences in continuous validated abstinence at 12 months were $11 \%, 4 \%, 8 \%(p=0.25)$. There was no significant difference between counselling alone and usual care, or in reduction in cigarette consumption between the treatment groups.

Conclusions: NRT given with brief counselling to hospital inpatients is an effective routine smoking cessation intervention.
\end{abstract}

S moking is the greatest preventable cause of mortality and morbidity in the developed world, ${ }^{12}$ and smoking cessation interventions involving behavioural support and nicotine or bupropion pharmacotherapy in primary care and in specialist cessation clinics are effective and highly cost effective..$^{3-5}$ However, the role of these interventions in patients admitted to hospital, many of whom are smokers, has not been clearly established. Intensive behavioural support has been shown to be effective in inpatients with cardiovascular disease $^{6-8}$ but is logistically difficult to deliver as a routine service to all smoking inpatients. Less intensive counselling would be more suitable for widespread application, but studies in unselected patients ${ }^{910}$ and patients with cardiovascular disease ${ }^{11}$ have found no evidence of benefit. Studies of nicotine replacement therapy (NRT) in hospital inpatients to date have been relatively small, and although suggestive of a beneficial effect, have not shown significant differences relative to placebo, ${ }^{12-15}$ results supported by a systematic review of the subject. ${ }^{16}$ We have therefore conducted a pragmatic open randomised controlled trial to determine whether a brief cessation counselling intervention suitable for widespread use, or the same counselling intervention given with NRT, is more effective than usual care in promoting smoking cessation in patients admitted to a UK teaching hospital.

\section{METHODS}

\section{Participants}

All medical and surgical admissions admitted to Nottingham City Hospital between March 1999 and April 2000 were screened by a research doctor or nurse within 48 hours of admission (see box 1 for eligibility criteria) and all current smokers-defined as those who described themselves as regular smokers and who had smoked their last cigarette within 28 days of admission-were invited to participate. All consenting individuals were enrolled and provided questionnaire details of smoking history, Fagerström test of nicotine dependence, ${ }^{17}$ smoking behaviour (Glover-Nilsson smoking behavioural questionnaire ${ }^{18}$ ), and completed the SF-36 quality of life questionnaire. ${ }^{19}$ Details of the main admission diagnosis and significant past medical history were recorded. Exhaled carbon monoxide was measured using a Bedfont Micro-Smokerlyzer (Bedfont Scientific Ltd, UK). The original protocol also proposed to use similar methods to recruit patients at St George's and King's College Hospitals in London; participation was low at these centres and recruitment was therefore abandoned at 3 and 8 months, respectively. The study was approved by the local research ethics committees at each hospital.

\section{Interventions}

Patients were randomised to one of three treatment groups following enrolment using a list generated for each centre, allocating equally in random permuted blocks of nine.

(1) Usual care: these patients had smoking status recorded but received no additional formal intervention.

(2) Counselling alone: a research doctor or nurse trained in smoking cessation counselling gave a 20 minute counselling 
Box 1 Eligibility criteria for patients

Inclusion criteria

- Male and female medical and surgical inpatients who were smokers whose last cigarette was within 28 days before admission.

- Able to provide written informed consent and understand English.

- 18 years of age or older.

- Expected to comply with the protocol.

- An acceptable level of consciousness.

- Expected duration of hospitalisation of at least 24 hours.

- Planning to remain at their current address for the next 12 months.

- Access to the telephone.

- Resident within a reasonable travelling distance from the hospital.

Exclusion criteria

- Pregnant (including suspected pregnancy), planned pregnancy, or breast feeding.

- Admission for psychiatric care.

- History of alcohol and/or illicit drug abuse in the last 12 months.

- Terminal illness (prognosis less than 12 months).

- Concurrent use of another investigational medication and within 1 month of entry into this study.

- Hypersensitivity towards nicotine or menthol.

- Previous enrolment into this study.

- Acute cerebrovascular accident.

session at the bedside and provided a written advice leaflet. Patients were advised of the effectiveness and availability of NRT.

(3) NRT plus counselling: a research doctor or nurse gave counselling as above and also offered a 6 week course of NRT. Patients were advised on the use of NRT and offered a choice of one of five different open labelled products (nicotine transdermal patch (15 mg, 16 hours), nicotine gum (2 or $4 \mathrm{mg})$, nicotine inhalator $(10 \mathrm{mg})$, nicotine sublingual tablet ( $2 \mathrm{mg}$ ), or nicotine nasal spray $(0.5 \mathrm{mg} / \mathrm{spray})$, Pharmacia Ltd, UK) which were then dispensed at the first visit and used according to product licence.

\section{Follow up}

All patients were asked whether they had smoked since enrolment, details of any adverse events were elicited, and exhaled carbon monoxide was measured immediately before discharge or at 7 days after enrolment, whichever was sooner. All patients were also invited to attend for review or interviewed by telephone 3 and 12 months after enrolment to ascertain whether they had smoked since their last visit, any adverse events, whether they had purchased any additional NRT, and to complete the SF-36 quality of life questionnaire. Exhaled carbon monoxide was measured in all patients who reported not smoking by home visit in those who were reviewed by telephone. Patients who did not attend their follow up appointments and/or could not be contacted after a minimum of two telephone calls were considered lost to follow up and assumed to be smoking.

\section{Sample size and power}

Our original power calculation assumed that each hospital would admit an average of four eligible smokers per day, yielding 540 patients per centre in 6 months and 1620 patients (540 patients in each treatment group) in total. Assuming that $5 \%$ of patients in usual care would not be smoking at 12 months, this sample size would have provided approximately $90 \%$ power at $5 \%$ significance to detect a doubling of the cessation rate to $10.2 \%$ at 12 months in the NRT plus counselling group. In the event the final sample size was lower than planned, mainly because the study was abandoned at two centres before completion of follow up.

\section{Analysis of data}

The primary outcome measure was continuous abstinence (defined as abstinence from any smoking from enrolment) validated by an exhaled carbon monoxide measurement of $<10$ parts per million ( $\mathrm{ppm}$ ) at discharge, 3 months and 12 months. Secondary outcome measures were point prevalence validated abstinence (defined as abstinence from any smoking since the previous visit) at discharge, 3 months and 12 months, reduction in cigarette consumption in those still smoking (measured as change from baseline in cigarettes per day) at 3 and 12 months, and quality of life at 3 and 12 months computed in terms of the eight dimensions of SF-36. ${ }^{19}$ Analysis was performed on an intention-to-treat basis using $\chi^{2}$ tests and risk ratios with $95 \%$ confidence intervals (CI) to compare abstinence at each follow up and, where significant, post hoc pairwise comparisons were performed. Absolute cigarette consumption in those who continued to smoke, measured as self-reported cigarettes per day, was compared between groups by the Kruskal-Wallis non-parametric test with post hoc pairwise comparisons using the Mann-Whitney U test.

\section{RESULTS}

\section{Baseline characteristics and rates of follow up}

A total of 1068 patients were screened at Nottingham City Hospital, of whom 274 (26\%) were eligible and consented to be enrolled, 92 to usual care, 91 to counselling alone, and 91 to NRT plus counselling (fig 1). Baseline characteristics (table 1) and admission diagnoses (table 2 ) did not differ significantly between the treatment groups. Of those randomised to receive counselling and NRT, 57 (63\%) chose a transdermal patch, 12 (13\%) chose an inhalator, $10(11 \%)$ chose gum, seven $(8 \%)$ chose sublingual tablets, and one ( $1 \%$ ) chose the nasal spray; four patients (4\%) declined to use any NRT. Reviews were carried out in the total patient population at discharge in 251 (92\%), at 3 months in $171(62 \%)$, and at 12 months in 112 (41\%, fig 1). Most of those lost to follow up did not attend their appointment and could not be contacted by telephone, although a small number were excluded after enrolment because they were discharged within 24 hours of admission.

\section{Abstinence from smoking and reduction in consumption}

Validated abstinence at discharge was significantly higher in the NRT plus counselling group than in the counselling alone or usual care groups $(55 \%, 43 \%$ and $37 \%$ respectively, $\mathrm{p}=0.045$, table 3 ) with a risk ratio (RR) for validated abstinence in those receiving NRT relative to those who were not of 1.38 (95\% CI 1.06 to $1.80, \mathrm{p}=0.018$ ). The difference between counselling alone and usual care was not significant. At 3 months abstinence remained highest in the NRT plus counselling group but the difference was not significant (table 3 ). Inclusion of the data available at discharge and at 3 months from the 123 participants recruited at the two London centres made no appreciable difference to these findings.

At 12 months abstinence rates were highest for the NRT plus counselling group and similar or lower in the counselling alone relative to the usual care group (table 3). The continuous validated abstinence rates for NRT plus counselling relative to counselling alone or usual care were $11 \%, 4 \%$, and $8 \%$, respectively $(\mathrm{p}=0.25)$, and for validated point abstinence $17 \%, 6 \%$ and $8 \%(\mathrm{p}=0.03)$. The RR for continuous validated abstinence for NRT plus counselling versus the other two groups combined was 1.83 (95\% CI 0.76 to $4.12, \mathrm{p}=0.15$ ), and for validated point abstinence the RR was 2.51 (95\% CI 1.25 to 5.03, $\mathrm{p}=0.009$ ). The effect of counselling alone was not significantly different from usual care (RR for validated point abstinence $0.58,95 \%$ CI 0.18 to $1.88, \mathrm{p}=0.4$ ). 


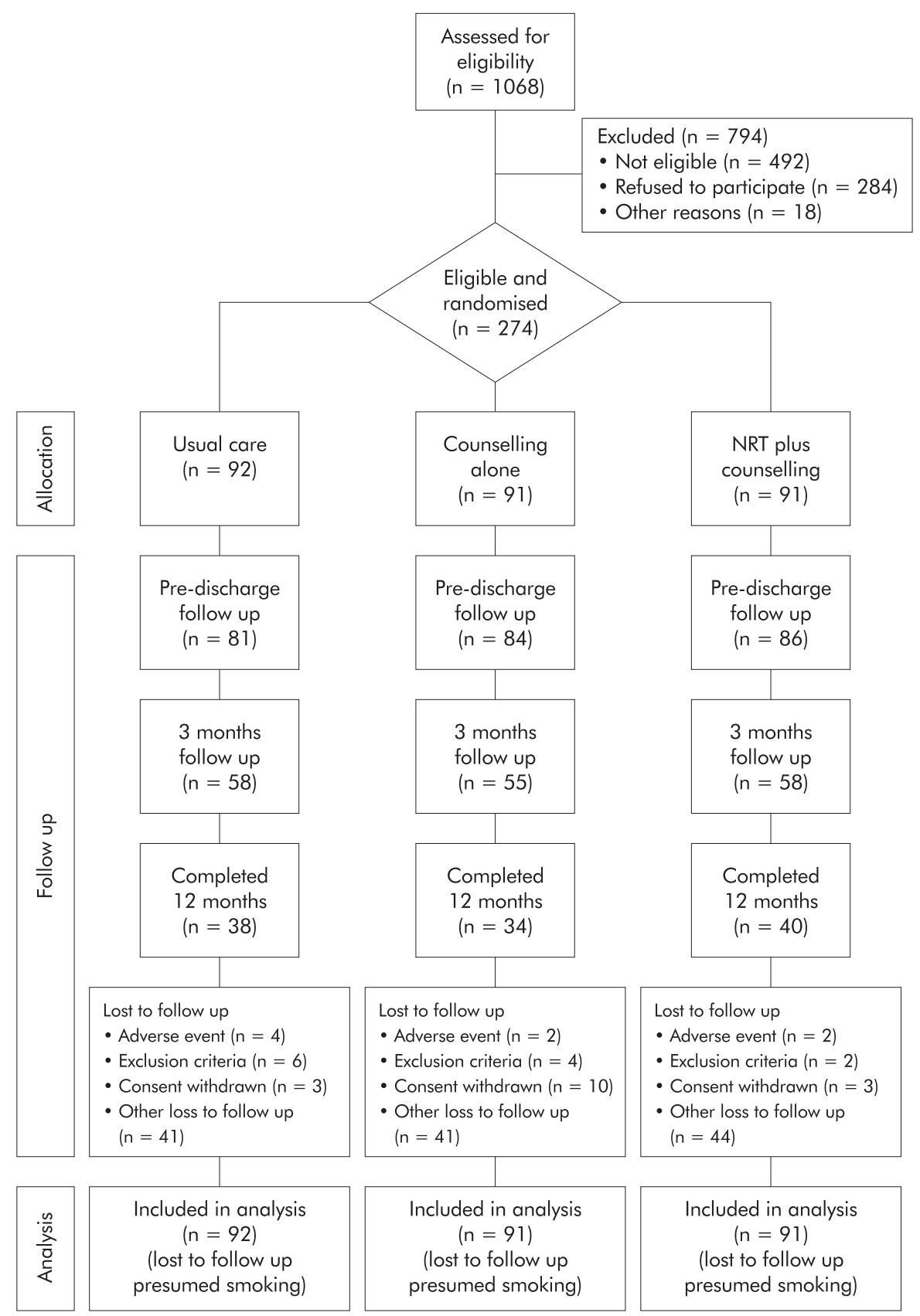

Figure 1 Flow of patients through study. NRT=nicotine replacement therapy.

\begin{tabular}{|c|c|c|c|c|}
\hline Baseline characteristics & $\begin{array}{l}\text { All patients } \\
(n=274)\end{array}$ & $\begin{array}{l}\text { Usual care } \\
(n=92)\end{array}$ & $\begin{array}{l}\text { Counselling } \\
(\mathrm{n}=91)\end{array}$ & $\begin{array}{l}\text { NRT }+ \text { counselling } \\
(n=91)\end{array}$ \\
\hline Sex (\% male) & $163(59.5)$ & $54(58.7)$ & $51(56.0)$ & $58(63.7)$ \\
\hline Mean (SD) age & $49.4(15.7)$ & $51.0(15.8)$ & $47.8(15.3)$ & $49.3(15.9)$ \\
\hline \multicolumn{5}{|l|}{ Ethnicity } \\
\hline White & $260(95.9)$ & $90(98.9)$ & $85(95.5)$ & 85 (93.4) \\
\hline Black & $10(3.7)$ & $1(1.1)$ & $4(4.5)$ & $5(5.5)$ \\
\hline Other & $1(0.4)^{\prime}$ & 0 & 0 & $1(1.1)$ \\
\hline \multicolumn{5}{|l|}{ Smoking history } \\
\hline Median (range) cigarettes/day & $17(1-80)$ & $15(1-60)$ & $20(3-40)$ & $20(3-80)$ \\
\hline Mean (SD) years smoked & $33.5(16.2)$ & $35.4(16.0)$ & $32.1(15.8)$ & $33.1(16.6)$ \\
\hline Median (IQR) FTND & $5(3-6)$ & $5(3-6)$ & $4(2-6)$ & $5(3-6)$ \\
\hline Mean (SD) Glover-Nilsson & $40.3(10.3)$ & $41.6(10.5)$ & $39.3(9.5)$ & $40.0(10.7)$ \\
\hline Any previous serious attempt to stop smoking, $n(\%)$ & $222(81.0)$ & $73(79.4)$ & $73(80.2)$ & $76(83.5)$ \\
\hline
\end{tabular}


Table 2 Main admission diagnosis classified by system in the treatment groups

\begin{tabular}{lllll}
\hline Main admission diagnosis & $\begin{array}{l}\text { Usual } \\
\text { care }\end{array}$ & Counselling & $\begin{array}{l}\text { NRT }+ \\
\text { counselling }\end{array}$ & Total, N (\%) \\
\hline Respiratory & 21 & 20 & 20 & $61(22.3)$ \\
Cardiac & 23 & 19 & 16 & $58(21.2)$ \\
Gastrointestinal & 16 & 17 & 16 & $49(17.9)$ \\
Renal and urinary & 7 & 9 & 9 & $25(9.1)$ \\
Musculoskeletal, connective tissue and bone & 2 & 7 & 6 & $15(5.5)$ \\
General & 5 & 5 & 4 & $14(5.1)$ \\
Reproductive and breast & 3 & 1 & 6 & $10(3.6)$ \\
Vascular & 3 & 2 & 3 & $8(2.9)$ \\
Neurological & 2 & 2 & 3 & $7(2.6)$ \\
Injury & 2 & 0 & 2 & $4(1.5)$ \\
Other systems & 7 & 9 & 6 & $22(8.0)$ \\
Unknown & 1 & 0 & 0 & $1(0.4)$ \\
Total & 92 & 91 & 91 & $274(100)$ \\
\hline NRT=nicotine replacement therapy. & \multicolumn{7}{|l}{} \\
\hline
\end{tabular}

Table 3 Number (\%) abstinent before discharge and at 3 and 12 months follow up

\begin{tabular}{|c|c|c|c|c|c|c|c|c|c|}
\hline \multirow[b]{2}{*}{ Follow up } & \multicolumn{3}{|c|}{ Self-reported point prevalence abstinence } & \multicolumn{3}{|c|}{ Validated point prevalence abstinence } & \multicolumn{3}{|c|}{ Validated continuous abstinence } \\
\hline & Usual care & Counselling & $\begin{array}{l}\text { NRT + } \\
\text { counselling }\end{array}$ & Usual care & Counselling & $\begin{array}{l}\text { NRT + } \\
\text { counselling }\end{array}$ & Usual care & Counselling & $\begin{array}{l}\text { NRT + } \\
\text { counselling }\end{array}$ \\
\hline Discharge & $39(42.4)$ & $43(47.3)$ & $55(60.4)^{*}$ & $34(37.0)$ & 39 (42.9) & $50(54.9)^{*}$ & - & - & - \\
\hline 3 months & $12(13.0)$ & 15 (16.5) & $18(19.8)$ & $10(10.9)$ & $9(9.9)$ & $15(16.5)$ & $10(10.9)$ & 7 (7.7) & $15(16.5)$ \\
\hline 12 months & $9(9.8)$ & $8(8.8)$ & $17(18.7)$ & $7(7.6)$ & $5(5.5)$ & $15(16.5) \dagger$ & $7(7.6)$ & $4(4.4)$ & $10(11.0)$ \\
\hline
\end{tabular}

Reduction in cigarette consumption at 3 months was greater in the NRT plus counselling group than in the counselling alone or usual care groups but not significantly so (mean (SD) reduction 8.3 (9.0), 6.3 (9.3) and 3.3 (9.9) cigarettes per day, respectively). There was no significant difference between the three treatment groups at 12 months $(\mathrm{p}=0.56)$.

\section{Quality of life}

All dimensions of quality of life were similar across the three treatment groups at baseline and at 3 months. The dimension measuring role limitation due to physical problems alone differed between treatment groups at 12 months $(p=0.05)$ with values on this score being better for those in the NRT plus counselling group than in the other two treatment groups.

\section{Adverse events}

There were 89 adverse events in a total of 65 patients, 33 of which were serious (three deaths and 30 other events, primarily due to complications of the admitting illness); there was no significant difference between the treatment groups. Five adverse events, none serious, were considered to be related to NRT (two skin rashes and one each of nausea, dizziness, and unpleasant taste).

\section{DISCUSSION}

This study shows that a smoking cessation package providing NRT with brief counselling significantly increases point prevalence abstinence at 1 year in hospital inpatients. The study also shows an increase of approximately twofold in continuous abstinence at 1 year that is consistent with the magnitude of the reported effect of NRT in outpatient and primary care populations, ${ }^{20}$ but was not statistically significant in the present study. The effect of counselling alone was not significantly different from usual care.

Although $26 \%$ of patients screened were subsequently randomised, there was a wider range of admission diagnoses than in previous studies of NRT in hospital inpatients, ${ }^{11-13} 1521$ and patients' cigarette consumption was typical of the national UK population, ${ }^{22}$ suggesting that our findings may be more generalisable to the UK population of inpatient smokers than previous work.

As part of a pragmatic approach, those patients randomised to the NRT plus counselling group were given a free choice of a range of nicotine products. While there is no evidence to suggest that any product is significantly better than another, ${ }^{23}$ patients overwhelmingly chose to use the transdermal patch which is the most widely purchased form of NRT in the UK ( F Nilsson, personal communication, 2002). A previous study of smokers' preferences for NRT products has shown a similar pattern. ${ }^{24}$ A small proportion (4\%) of those randomised to counselling and NRT chose to refuse it, which may have distorted the true effect of NRT. Only a minority of patients obtained additional NRT after discharge, and the numbers did not vary significantly between treatment groups (data not shown). This study was not placebo controlled because we aimed to conduct a pragmatic study of the effectiveness of the routine delivery of a package of NRT and brief counselling, rather than to test the efficacy of NRT.

Our finding that counselling alone had no effect on smoking cessation in these patients conflicts with current evidence on the efficacy of similar interventions in clinical contexts outside hospital. ${ }^{25}$ This finding may be due to low study power, but it is possible that the lack of effect was due to the relatively brief nature of the counselling given, or to the fact that no follow up support was offered since counselling involving greater contact is associated with greater cessation rates. ${ }^{4}$ However, an alternative inference, which is supported by evidence from a systematic review of other hospital based trials that smoking cessation counselling without subsequent follow up is ineffective, ${ }^{16}$ is that brief counselling simply does not work in hospital inpatients. Further studies are necessary to determine whether more sustained behavioural support is more effective in this context.

This is the first randomised controlled trial to show the effectiveness of NRT measured as point prevalence abstinence at 12 months in smokers admitted to hospital. In contrast with 
other studies of inpatient therapy in restricted disease subgroups, ${ }^{11-13} 1521$ this study has addressed the effectiveness of routine service provision to patients admitted to hospital regardless of diagnosis, and suggests that NRT given in association with brief counselling is effective, although further larger studies are needed to confirm whether there was a real effect on continuous abstinence. Subgroup analysis of our results revealed a similar effect of NRT with brief counselling in patients with and without smoking related diseases (data not shown). Our findings are in contrast to a recent paper in which Hand and colleagues showed that a 3 week course of NRT given in conjunction with intensive counselling (four sessions lasting up to 2.5 hours in total) did not significantly increase cessation rates over counselling alone in hospital patients with smoking related disease who were willing to see a counsellor. ${ }^{15}$ Possible reasons for the failure of NRT in this study include the restriction to patients with smoking related diseases, a group which may be less successful at stopping smoking than other hospital patients, ${ }^{27-29}$ that NRT may not be effective in hospital patients, and that the course of NRT given may have been too short, although the 1 year cessation rate in the counselling group was $14 \%$ compared with $7 \%$ in our study, suggesting that greater counselling input was more effective. Our findings, taken in conjunction with those of Hand et al, suggest that either NRT or intensive counselling are similarly effective in hospital patients, but that NRT plus brief counselling is a more practically applicable approach. We conclude that, in the absence of contraindications, NRT should be given with brief counselling as a routine therapy to all smokers admitted to hospital who indicate that they are prepared to try to stop smoking.

\section{Authors' affiliations}

A Molyneux, S Lewis, U Leivers, A Anderton, M Antoniak, J Britton Division of Respiratory Medicine, University of Nottingham, City Hospital Nottingham, UK

J Moxham, Guy's, King's and St Thomas' School of Medicine, London,

UK

A Brackenridge, A McNeill, R West, Department of Psychology, St

George's Hospital Medical School, London, UK

F Nilsson, Pharmacia Consumer Healthcare, Medical Affairs, Helsingborg, Sweden.

This study was supported by a grant from Pharmacia Consumer Healthcare, Helsingborg, Sweden and was instigated with the support of the Health Education Authority, UK.

\section{REFERENCES}

1 Tobacco Advisory Group of the Royal College of Physicians. Tobacco smoking in Britain: an overview. In: Nicotine addiction in Britain. London: Royal College of Physicians, 2000: 1-26.

2 Tobacco use-United States, 1900-1999. MMWR 1999:48:986-93.

3 Parrott S, Godfrey C, Raw M, et al. Guidance for commissioners on the cost effectiveness of smoking cessation interventions. Thorax 1998;53(Suppl 5 Pt 2):S1-38

4 West R, McNeill A, Raw M. Smoking cessation guidelines for health professionals: an update. Thorax 2000;55:987-99.

5 Tobacco Use and Dependence Clinical Practice Guideline Panel, Staff, and Consortium Representatives. A clinical practice guideline for treating tobacco use and dependence: a US Public Health Service report. JAMA 2000;283:3244-54.

6 DeBusk RF, Miller NH, Superko HR, et al. A case-management system for coronary risk factor modification after acute myocardial infarction. Ann Intern Med 1994:120:721-9.

7 Miller NH, Smith PM, DeBusk RF, et al. Smoking cessation in hospitalized patients. Results of a randomized trial. Arch Intern Med 1997; 157:409-15.

8 Taylor CB, Houston-Miller N, Killen JD, et al. Smoking cessation after acute myocardial infarction: effects of a nurse-managed intervention. Ann Intern Med 1990:1 13:1 18-23.

9 Rigotti NA, Arnsten JH, McKool KM, et al. Efficacy of a smoking cessation program for hospital patients. Arch Intern Med 1997; 157:2653-60.

10 Stevens VJ, Glasgow RE, Hollis JF, et al. Implementation and effectiveness of a brief smoking-cessation intervention for hospital patients. Med Care 2000;38:451-9.

11 Hajek P, Taylor TZ, Mills P. Brief intervention during hospital admission to help patients to give up smoking after myocardial infarction and bypass surgery: randomised controlled trial. BM 2002;324:87-9.

12 Campbell IA, Prescott RJ, Tjeder-Burton SM. Smoking cessation in hospital patients given repeated advice plus nicotine or placebo chewing gum. Respir Med 1991;85: 155-7

13 Campbell IA, Prescott RJ, Tjeder-Burton SM. Transdermal nicotine plus support in patients attending hospital with smoking-related diseases: a placebo-controlled study. Respir Med 1996;90:47-51.

14 Lewis SF, Piasecki TM, Fiore MC, et al. Transdermal nicotine replacement for hospitalized patients: a randomized clinical trial. Prev Med 1998;27:296-303.

15 Hand S, Edwards S, Campbell IA, et al. Controlled trial of three weeks nicotine replacement treatment in hospital patients also given advice and support. Thorax 2002;57:715-8

16 Munafo M, Rigotti N, Lancaster T, et al. Interventions for smoking cessation in hospitalised patients: a systematic review. Thorax 2001:56:656-63

17 Heatherton TF, Kozlowski LT, Frecker RC, et al. The Fagerstrom test for nicotine dependence: a revision of the Fagerstrom tolerance questionnaire. Br J Addict 1991;86:1119-27.

18 Glover ED, Nilsson F, Westin A. The Glover-Nilsson Smoking Behavioural Questionnaire (GN-SBQ). Society for Research on Nicotine and Tobacco 3rd European Conference, Paris, September 2001, poster presentation.

19 Jenkinson C, Layte R, Wright L, et al. The UK SF-36: an analysis and interpretation manual. Oxford: Health Service Research Unit, University of Oxford, 1996.

20 Silagy C, Lancaster T, Stead L, et al. Nicotine replacement therapy for smoking cessation. Cochrane Database Syst Rev 2001 ; CD000 146.

21 Joseph AM, Norman SM, Ferry LH, et al. The safety of transdermal nicotine as an aid to smoking cessation in patients with cardiac disease. N Engl J Med 1996:335:1792-8.

22 Office for National Statistics. Statistics on smoking in England, 1976-1996. London: The Stationery Office, 1998

23 Hajek P, West R, Foulds J, et al. Randomized comparative trial of nicotine polacrilex, a transdermal patch, nasal spray, and an inhaler. Arch Intern Med 1999:159:2033-8.

24 West R, Hajek P, Nilsson F, et al. Individual differences in preferences for and responses to four nicotine replacement products. Psychopharmacology (Berl) 2001;153:225-30.

25 Rice VH, Stead LF. Nursing interventions for smoking cessation. Cochrane Database Syst Rev 2001;CD001 188

26 Silagy C, Stead LF. Physician advice for smoking cessation. Cochrane Database Syst Rev 2001;CD000165

27 Subcommittee of the Research Committee of the British Thoracic Society. Comparison of four methods of smoking withdrawal in patients with smoking related diseases. BMU (Clin Res Ed) 1983;286:595-7.

28 British Thoracic Society. Smoking cessation in patients: two further studies by the Research Committee of the British Thoracic Society. Thorax 1990;45:835-40.

29 Foulds J, Stapleton J, Hayward M, et al. Transdermal nicotine patches with low-intensity support to aid smoking cessation in outpatients in a general hospital. A placebo-controlled trial. Arch Fam Med 1993;2:417-23. 